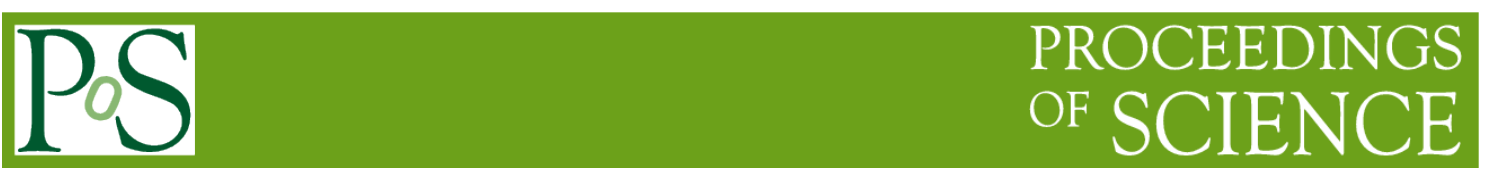

\title{
Multi-tomography of polars from SALT spectroscopy
}

\author{
Stephen B. Potter ${ }^{1}$ \\ South African Astronomical Observatory \\ Cape Town, South Africa \\ E-mail:sbp@saao.ac.za
}

\section{Enrico J. Kotze}

South African Astronomical Observatory and

Astrophysics, Cosmology and Gravity Centre (ACGC), Department of Astronomy, University of Cape Town,

Cape Town, South Africa

E-mail: ejk@saao.ac.za

\section{Vanessa A. McBride}

South African Astronomical Observatory and Astrophysics, Cosmology and Gravity Centre (ACGC), Department of Astronomy, University of Cape Town,

Cape Town, South Africa

E-mail:vanessa@saao.ac.za

Two highlights of SALT observations of the polar UZ For are presented; the first used the high-speed photometry mode of SALTICAM to measure accurate eclipse times from which 2 extra-solar planets are inferred. The second used the long-slit spectroscopy mode of RSS to obtain phase-resolved spectroscopy in order to perform detailed investigations of the accretion flow; also in the polar UZ For.

SALT Science Conference 2015

1-5 June 2015

Stellenbosch Institute of Advanced Study, South Africa

\section{${ }^{1}$ Speaker}

(C) Copyright owned by the author(s) under the terms of the Creative Commons 


\section{Introduction}

The study of Cataclysmic Variables (CVs) in general not only advances our understanding of this class of object in their own right, but also provides the opportunity to explore a broad range of astrophysical phenomena, the most obvious being accretion. CVs have been pivotal to the development of accretion disc theory. This is because they are nearby (and hence bright), evolve on short time-scales (hours to weeks) and are therefore ideal for indirect micro-arcsecond imaging techniques such as Doppler tomography from which one can probe the binary constituents (the stream, disc, secondary and white dwarf). Furthermore the evolution of binary systems leads to some of the most exotic inhabitants of our Galaxy, such as the black-hole binaries, supernovae and even the further distant gamma-ray bursters. CVs are a vital link in the evolutionary chain of binary stars, coming immediately after the common-envelope phase and evolving via magnetic braking and gravitational radiation. Consequently observations of CVs have played a key role in the development of these theories.

The additional ingredient of a magnetic field in polars adds a further dimension to the exploration of accretion. Polars are a subclass of magnetic CVs (mCVs) in which the white dwarf has a strong magnetic field and rotates synchronously with the orbital period. They are a source of hard and soft X-rays and cyclotron optical emission. This is because the accretion material is eventually magnetically channeled onto the surface of the white dwarf (WD) where a small shock region forms. The material in the shock becomes highly ionized, very dense and very hot (several $10 \mathrm{keV}$ ), leading to the emission of X-ray radiation (by Bremsstrahlung) and cyclotron radiation (optically polarized). With orbital periods of a few hours we effectively get almost a 360 degree view of the $\mathrm{CV}$ over the orbit, thus enabling the use of multiple tomographic techniques.

Eclipsing polars provide the best opportunity to exploit these tomographic techniques. In Section 2 we describe how we have made use of their eclipsing nature to obtain accurate eclipse timing measurements that show cyclic departures from a linear ephemeris. In Section 3 we describe our new development of the Doppler tomographic technique to obtain new insights from orbitally phase-resolved spectroscopy of the same system. 


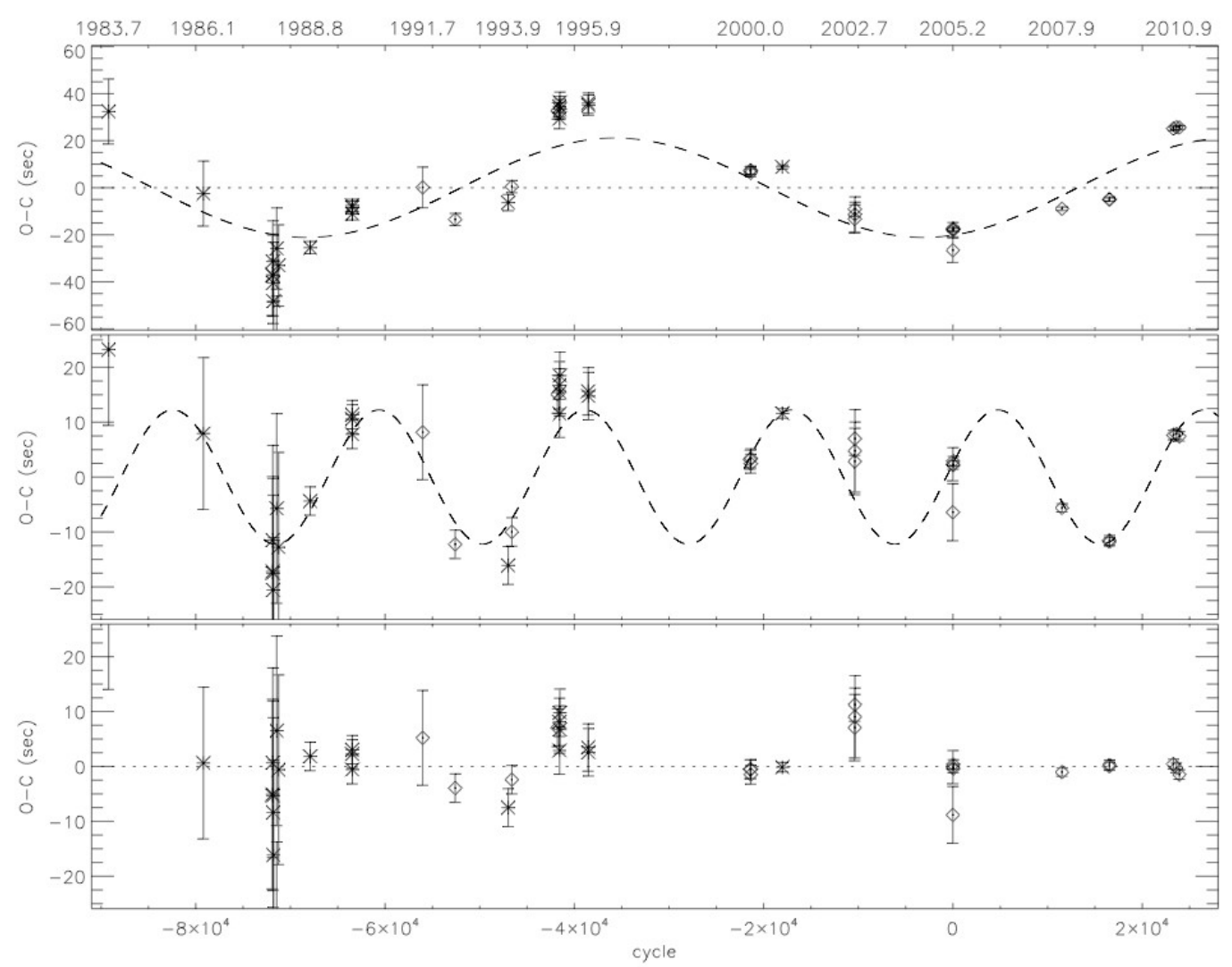

Figure 1. (extracted from [1]) shows that there are departures in the eclipse times of UZ For from a simple quadratic ephemeris of up to $\sim 60$ s (top panel). Middle and bottom panels show the O-C after subtraction of the first and second elliptical terms, corresponding to periods of 5.25(25) and 16(3) years respectively.

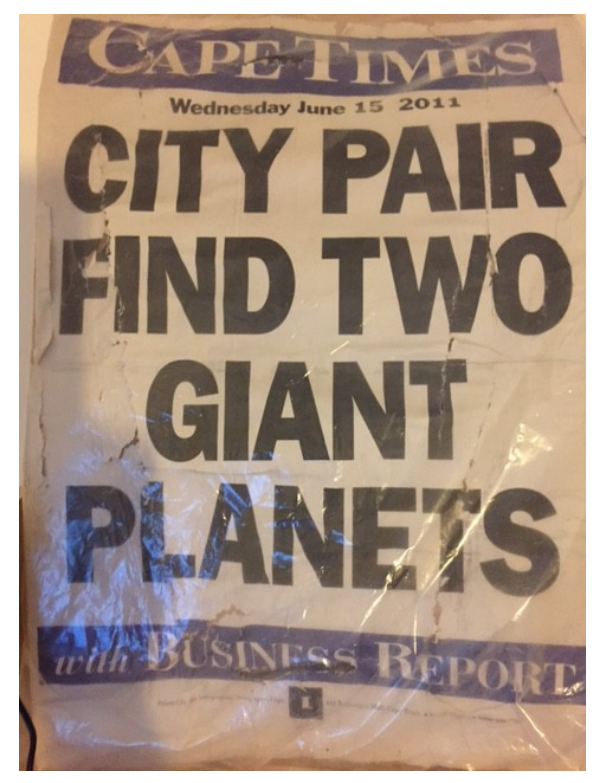

Figure 2. Local Cape Town road signage on June 15, 2011. 


\section{Possible detection of two giant extrasolar planets orbiting the eclipsing polar UZ Fornacis}

[1] combined accurate eclipse timing measurements, made with SALTICAM, with archived measurements from other observatories spanning almost 30 years. Figure 1 (extracted from [1]) shows that there are departures in the eclipse times of UZ For from a simple quadratic ephemeris of up to $\sim 60 \mathrm{~s}$. The departures are suggestive of two periodicities of $\sim 16$ and $\sim 5.25$ years. The two favoured mechanisms to drive the periodicities are either two giant extrasolar planets as companions to the binary or a magnetic cycle mechanism (e.g. Applegate's mechanism; [2]) of the secondary star. However, Applegate's mechanism would require the entire radiant energy output of the secondary and therefore would seem to be the least likely of the two, barring any further refinements in the effect of magnetic fields (e.g. [3]). A two planet model is also problematic given the quality of the data in that a high eccentric orbit, for at least one of the planets, seems to be required to fully capture all of the eclipse times. High eccentricities are unlikely to produce dynamically stable orbits.

As yet there is insufficient data on UZ For to identify conclusively the mechanism responsible for the periodic changes in its eclipse times, and indeed more than one mechanism could be present. Further good signal/noise, high time resolved observations of UZ For and other similarly eclipsing systems are encouraged.

Meanwhile, these so called extra-solar planets are currently a hot area of astronomical research and a few thousand have now been discovered over the past few years attracting popular press coverage. Indeed this particular work enjoyed local South African popularity with TV, radio and newspaper interviews. E.g. Figure 2 depicts the local road signage on the day the work was accepted for publication; the "CITY PAIR" being the first two authors of [1].

\section{Inside-out Doppler tomography of the eclipsing polar UZ Fornacis}

[4] developed Doppler tomography as a technique aimed at constructing a two-dimensional velocity image (tomogram) of the accretion discs of CVs using an emission line in its spectra sampled at a number of orbital phases. Doppler tomography has revolutionised the interpretation of orbitally phase-resolved spectroscopic observations of interacting binary systems. It has become a valuable tool for resolving the distribution of line emission in CVs and other binary systems.

Recently we [5] presented our investigation of reversing the velocity axis in order to create an inside-out Doppler coordinate framework with the intent to "correct" the inside-out appearance of Doppler tomograms. We showed that the projection of the accretion disc in velocity space now appears correctly orientated with the inner edge close to the maximum velocity origin and its outer edge closer to the zero-velocity outer circumference. Furthermore, the gas stream and secondary star are projected on the outside of the disc with the bright spot of the stream-disc impact region on the disc's outer edge in the inside-out velocity space. Our tests also show that there is a redistribution of the relative brightness of emission components throughout the tomograms, i.e., where the standard framework tends to concentrate and enhance lower velocity 
features towards the origin, the inside-out velocity framework tends to concentrate and enhance higher velocity features towards the origin. Conversely, the standard framework disperses and smears the higher velocities farther away from the origin whereas the inside-out framework disperses and smears the lower velocities.

We also applied our inside-out technique to magnetic CVs. Figure 3 shows our preliminary results applied to the same system investigated in Section 2; UZ For. Spectroscopic observations were obtained with the RSS on SALT over several nights in order to cover one complete orbit. Careful planning, using the PIPT tools, was required in order to avoid repeated observations of the same orbital phases.

The trailed HeII (4686) spectrum clearly shows multiple components displaying the familiar Doppler variations over the orbital period. Figure 3 (upper right) shows the observations mapped on the "normal" Doppler coordinate frame with a simple mCV model overplotted. Even though the trailed spectra show multiple components, the Doppler tomogram is somewhat disappointing in that the emission seems to be mostly concentrated towards the center of the image. Also, the high velocity wing component from the emission line is washed out in the Doppler image.

The lower right panel shows the observations mapped onto out inside-out Doppler coordinate frame. This coordinate frame has clearly mapped out the three main emission line components into velocity space. Emission from the secondary, the ballistic stream and in particular the accelerating magnetically confined accretion flow are well defined.

\section{Summary and future work}

With high-speed SALTICAM photometry and phase-resolved RSS spectroscopy we have successfully demonstrated SALT's ability to perform detailed single object studies of mCVs. Through high-speed photometry we have potentially discovered extra-solar planets and through spectroscopy we have effectively mapped the velocity components in the magnetic CV UZ For.

We next plan to extend the inside-out Doppler tomography technique to investigate the velocity components of the accretion flow in intermediate polars. These are probably weaker field mCVs, where the WD is spinning asynchronously to the orbit at typically $\sim 1000 \mathrm{~s}$. We will employ spin tomograms which uses spectroscopic observations phase-folded on the white dwarf spin period (rather than the orbital period) to obtain velocity maps of the magnetically confined accretion curtains in these systems.

Furthermore, phase-folded spectroscopic observations also contain "phase" information in addition to Doppler velocities. We are currently investigating how the phase information can be extracted to provide further detailed insights into the accretion dynamics of CVs. 

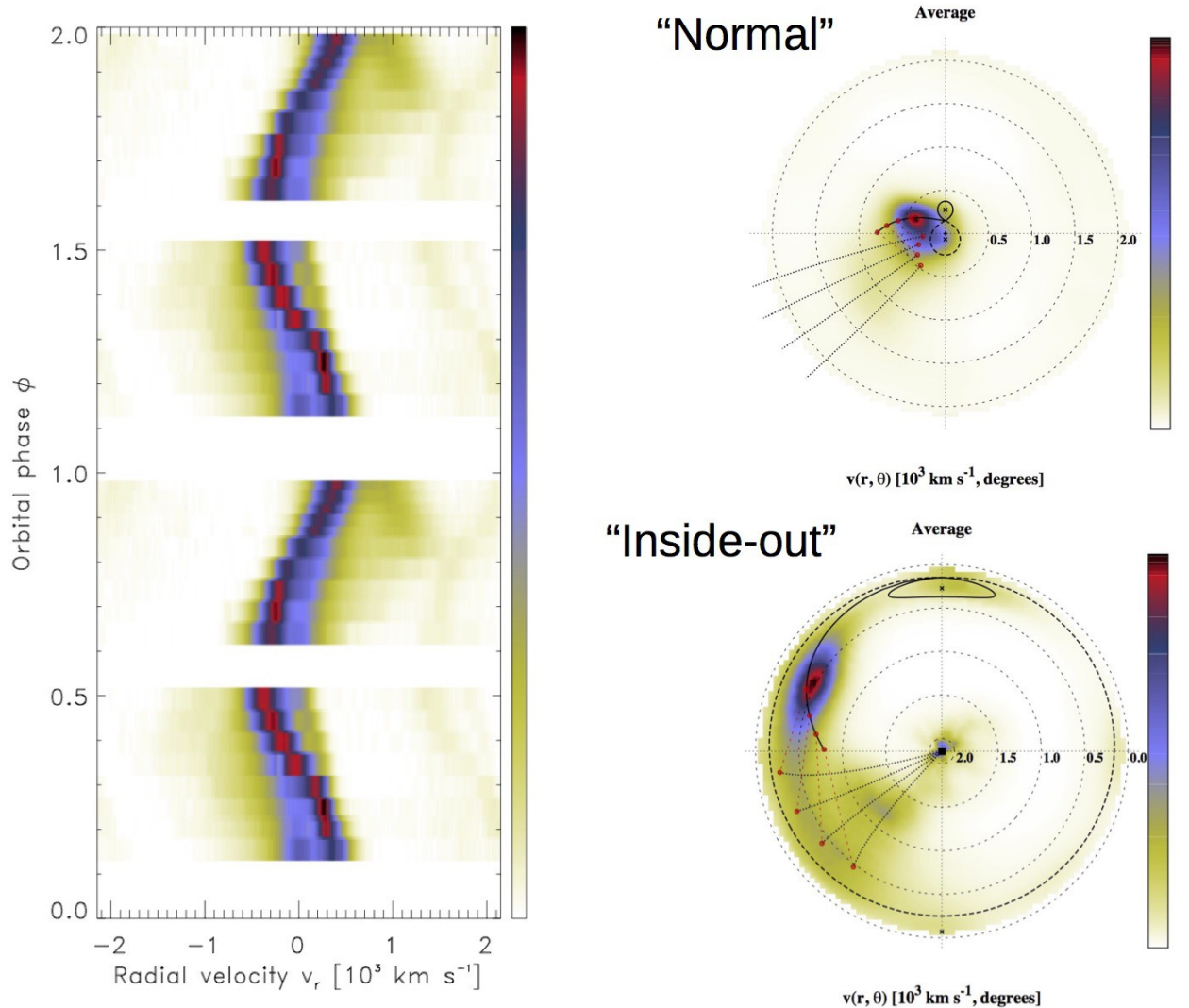

Figure 3. Trailed spectra of the polar UZ For and corresponding Doppler tomograms; see text for details.

\section{References}

[1] Potter et al. Possible detection of two giant extrasolar planets orbiting the eclipsing polar UZ Fornacis, 2011, MNRAS, 416, 2202.

[2] Applegate J. H., A mechanism for orbital period modulation in close binaries, 1992, ApJ, 385, 621

[3] Lanza A. F., Rodono M., Rosner R., Orbital period modulation and magnetic cycles in close binaries, 1998, MNRAS, 296, 893

[4] Marsh T. R., Horne K., Images of accretion discs. II - Doppler tomography, 1988, MNRAS, 235, 269

[5] Kotze E. J., Potter S. B., McBride V. A., Exploring inside-out Doppler tomography: non-magnetic cataclysmic variables, 2015, A\&A, 579, 77 